\title{
The Havoc of a Giant Spleen
}

\section{Summary}

We present a case of a 50-year-old female with previously diagnosed Felty's syndrome who experienced a number of complications related to massive splenomegaly including cytopenias. The patient required multiple hospitalizations during which a splenic infarction and splenothoracic fistula were discovered. Ultimately, the patient underwent splenectomy which was further complicated by post-operative multi-vein thrombosis. Analysis of this patient provides an illustration of the potential for significant morbidity and mortality associated with the often ignored finding of splenomegaly that is rarely reported in current literature.

\section{Background}

Felty's syndrome (FS) is the triad of rheumatoid arthritis (RA), neutropenia (absolute neutrophil count $<2000 / \mu \mathrm{L}$ ), and splenomegaly [1]. Although splenomegaly is included in the triad, most resources agree that splenomegaly is not an absolute diagnostic requirement. The lifetime risk of developing FS for a patient diagnosed with RA has been estimated at approximately 1\% [2]. Most commonly, FS is seen in patients with RA who develop neutropenia approximately ten years after initial presentation. The diagnosis of FS is one of exclusion and before the official diagnosis is made, one must rule out other aetiologies of neutropenia and splenomegaly.

For patients with recurrent infections and neutropenia, splenectomy should be considered [3]. Neutropenia will improve in $60-70 \%$ of post-splenectomy patients but relapse of neutropenia as well as recurrent infections in the presence of normal neutrophil counts may occur $[2,4]$.

It is important to note that splenectomy can lend to complications as well. Platelet counts after splenectomy have been shown to increase $30-100 \%$. Post-splenectomy reactive thrombocytosis has an incidence of $75-82 \%$ with peak platelet counts typically reached within two weeks [5]. Common complications of thrombocytosis are thrombosis and hemorrhage [5]. Post-splenectomy venous thrombosis happens most commonly when platelet counts reach $600-800 \mathrm{~K} / \mu \mathrm{L}$ and occurs in approximately $5 \%$ of patients [6].

\section{Case Presentation}

A 50 year-old woman with a history of Felty's Syndrome, currently treated with methotrexate, presented to the emergency department with a chief complaint of abdominal pain and fever. The patient was diagnosed with RA approximately 9 years ago and had been attempted to be treated with biologic therapy over her treatment course. Most recently she has been on etanercept, but with worsening pancytopenia requiring transfusions therapy was de-escalated to methotrexate alone. The patient was hospitalized multiple times in the recent past for neutropenic fever and recurrent infections with imaging indicative of splenic infarction and stable splenomegaly (See Figure 1).

On the day of presentation, the patient complained of severe, diffuse abdominal pain with associated anorexia, nausea, bilious

\section{Journal of}

Clinical \& Medical Case Reports

\section{Amanda J. Finley ${ }^{1 *}$, Lindsey Mary Beth ${ }^{2}$, Griffin Mary $^{2}$ and Griffin Daniel ${ }^{2}$}

${ }^{l}$ Nova Southeastern University, Magnolia Regional Health Center, USA

${ }^{2}$ Graduate Medical Education, Magnolia Regional Health Center, USA

Address for Correspondence:

Amanda J. Finley, DO, Nova Southeastern University, Magnolia Regional Health Center, Corinth, MS 38834, USA, Tel: 662-396-1286; Fax: 844-3415518; E-mail: afinley@osteo.wvsom.edu

Submission: 07 June, 2016

Accepted: 14 June, 2016

Published: 20 June, 2016

Copyright: $\odot 2016$ Finley AJ, et al. This is an open access article distributed under the Creative Commons Attribution License, which permits unrestricted use, distribution, and reproduction in any medium, provided the original work is properly cited.

Reviewed \& Approved by: Dr. Shan He, Fels Institute of Cancer Research and Molecular Biology, Temple University, USA

vomiting and diarrhea. She appeared to be in moderate distress with moderate-to-severe diffuse abdominal pain, greatest in the left upper quadrant, without rebound or guarding. Massive splenomegaly noted several centimeters below the costal margin into the left lower quadrant and was also noted to cross the midline. Auscultation of the lungs revealed decreased air movement bilaterally to the bases with inspiratory crackles in the left lung base. CT of the abdomen and pelvis revealed increased, severe splenomegaly with a linear area of decreased attenuation in the posterior spleen, indicative of splenic infarction and a moderate-sized left pleural effusion (Figure 2). Laboratory results were within normal limits with the exception of neutropenia with an absolute neutrophil count of $360 / \mu \mathrm{L}$.

After surgical evaluation, CT imaging was reviewed with radiology. Although not originally noted, there was concern for a splenothoracic fistula to the left thoracic cavity in addition to persistent splenomegaly and splenic infarction (See Figure 2). It was determined to proceed

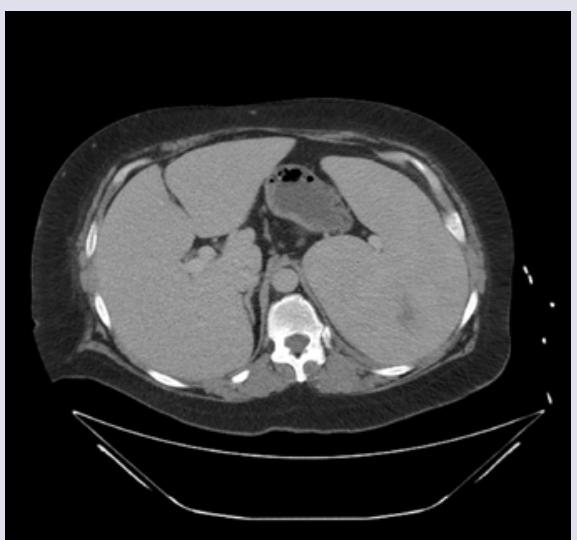

Figure 1: CT comparing spleen and liver size. 


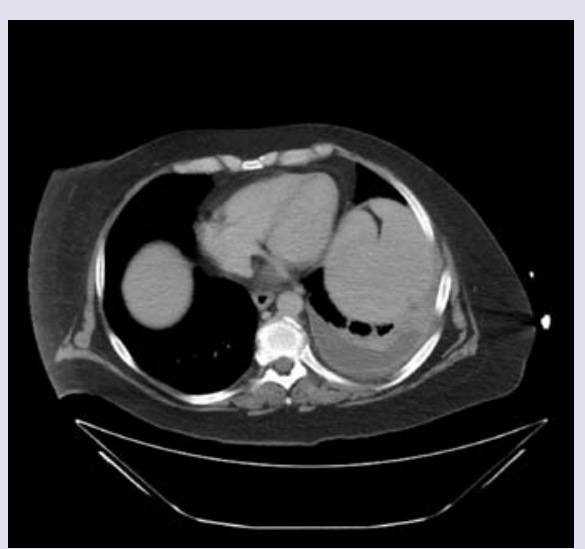

Figure 2: Left pleural effusion, question of radiographic fistula.

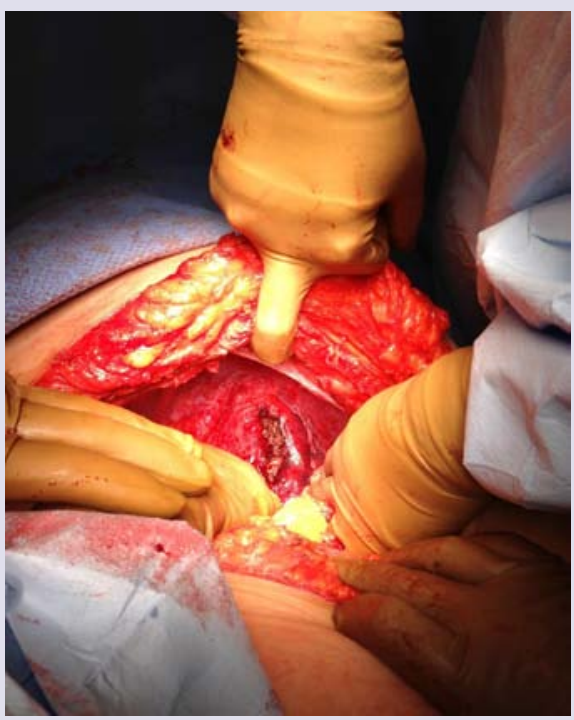

Figure 3: Intraoperative Spleen.

with splenectomy and further exploratory laparotomy secondary to recurrent infections, abdominal pain and findings on radiologic imaging. Patient was placed on empiric cefepime due to neutropenia at the time of presentation.

Open laparotomy revealed massive splenomegaly with area of infarction and necrosis as well as dense adhesions present on the superior pole of the spleen where the diaphragm met the chest wall (See Figure 3). The spleen was successfully removed, leaving a cavity as well as an incomplete splenothoracic fistula. Cultures obtained did not reveal any infectious aetiologies. Cardiothoracic surgery evaluated the patient while in the operating room and it was determined that no further surgical intervention was indicated and the operation was completed without complications. The patient was placed on empiric trimethoprim-sulfamethoxazole for 10 days postoperatively as well as aspirin $81 \mathrm{mg}$ for thromboembolic prophylaxis and the patient was discharged.

Several weeks after surgery, the patient was again seen in the emergency department with postprandial abdominal pain, nausea, vomiting, anorexia and progressively worsening weakness. Laboratory results were significant for thrombocytosis with platelets of $884 / \mu \mathrm{L}$ and anemia with hemoglobin of $9 \mathrm{~g} / \mathrm{dL}$ and hematocrit of $29 \%$. Patient reported good compliance with daily aspirin prophylaxis. CT of the abdomen revealed thrombosis involving the portal vein, superior mesenteric vein and splenic vein with stranding suggestive of vascular congestion. The patient was placed on Heparin for anticoagulation and appropriately bridged to warfarin therapy. Plateletpheresis and thrombectomy were considered but it was deferred due to significant clinical improvement with medical maximization. She was appropriately stabilized with close monitoring of INR and discharged home for later de-escalation to aspirin alone. Of note the patients' neutropenia had resolved following splenectomy.

\section{Treatment}

Post-splenectomy, the patient completed a course of trimethoprimsulfamethoxazole for anti-bacterial prophylaxis. Operative cultures remained negative. She was discharged home on aspirin for thrombosis prophylaxis. Despite aspirin therapy, she experienced severe thrombotic complications. She was therefore appropriately treated with anticoagulation therapy using warfarin. After several months on warfarin she was transitioned to aspirin which she will continue for lifelong thrombosis prophylaxis. The patient continues to be monitored by her rheumatologist and haematologist for treatment of her RA.

\section{Outcome and Follow-Up}

Three months post-splenectomy, the patient shows improved neutropenia and thrombocytosis. Although she had prolonged documented neutropenia, an infectious etiology for the necrotic region seen on radiography was never identified. She has been hospitalized once since her thrombotic event due to intractable abdominal pain but has not had any further serious complications.

The pathological review 2291 gram spleen that is $32 \times 23 \times 5.5 \mathrm{~cm}$. There is a capsule laceration that is $8 \mathrm{~cm}$ in length $\mathrm{x} 1.5 \mathrm{~cm}$ wide. The remainder of the capsule is relatively unremarkable. The hilum has a congested blood vessel as well as multiple firm nodules that appear to represent lymph nodes. They are 1.2 and $1.5 \mathrm{~cm}$ in greatest dimension respectively. On sectioning the spleen, it is markedly congested and no tumors or cysts are present. The spleen shows marked red pulp expansion with hemorrhage, histiocytes, and a lymphocyte population. By immunohistochemical staining, the lymphocytes are most consistent with T-cell large granular lymphocytes (LGLs--CD3, CD8, CD7, TIA-1 and granzyme positive, negative for CD57). These cells show slightly decreased CD5 expression but no co-expression of CD57. Histiocytes are also increased within the red pulp and there is extramedullary hematopoiesis. The white pulp is largely unremarkable. Overall, the features identified within this spleen are within the spectrum of those commonly observed within Felty syndrome patients and is consistent with a benign large granular lymphocytosis. No distinctly aberrant population consistent with a T-LGL leukemia is identified. The post-splenectomy CBC white count shows resolution of the leukopenia and neutropenia and further supports the likely benign T-cell population. Close clinical follow up of peripheral blood counts showed no reoccurrence of neutropenia and follow up bone marrow biopsy has been deferred. 


\section{Discussion}

Splenomegaly can arise from several etiologies including congestive, infiltrative, hypersplenic engorgement in haematological conditions, congenital defects, malignant processes, or chronic inflammation as was suspected in our patient with RA [7]. The complications for splenomegaly are also equally numerous but splenectomy needs to be considered with severe pain and significant cytopenias. Our patient did experience significant sequelae from her splenectomy including pain, cytopenias, infarction, necrosis fistulization.

Of particular interest is the question if there was fistulisation between the area of splenic necrosis and the left pleural space as was suggested on radiological imaging and pathological examination. Research on splenothoracic fistulas and associated conditions is very limited; however, it can be concluded that splenothoracic fistula formation requires a specific set of pathological processes before development. These processes include splenomegaly or a cause for splenic enlargement and superior splenic pole infection, infarction, or abscess development $[3,8,9]$. Evidence of this condition was found in only two cases that involved hydatid cyst of the spleen that resulted in fistula formation as well as a case involving a splenic pseudocyst with likely underlying malignancy that formed an abscess and fistulized through the diaphragm $[8,9]$.

Learning points from this case can be especially emphasized from the management of the complications of her splenectomy in relation to her reactive thrombocytosis and multivessel thrombosis. Splenectomy in patients with various hematologic has been associated with an increased incidence of vascular complications, including venous and arterial thrombosis and pulmonary hypertension $[6,10]$. The marked thrombocytosis following splenectomy was thought to be the main contri0butor of her multi-vessel thrombosis. Current management suggests that antiplatelet therapy only should suffice but if the patient experiences thrombosis, it has been shown that anticoagulation with unfractionated or low-molecular weight heparin followed by oral anticoagulation is the most effective treatment [11]. While this has been shown effective, there are currently no standardized protocols in place to indicate appropriate post-surgical prophylaxis or length of anticoagulation post-thrombotic event [11]. The patient was without family history of clotting or history of obstetrical losses. It is unclear if the splenic infarction was a result of a hypercoagulable process or if could be contributed to the marked splenomegaly alone. Of note the patient did have persistent presence in the serum of at least one type of antiphospholipid antibody, specifically lupus anticoagulant. Further follow up with hematology should rule out any other hypercoagulable states that would indicate lifelong anticoagulant therapy.

The patient presented to us at a point where she was already diagnosed with Felty's syndrome and had failed several attempts at biologic therapy. Patient had marked neutropenia but it is not known from our standpoint how much the biologic therapy had contributed to her reoccurring bouts of neutropenia and infections and how much could be attributed to the granulocyte destruction associated with Felty's. Review of pathological examination found the features identified within the spectrum of those commonly observed within Felty's syndrome patients and is consistent with a benign large granular lymphocytosis. "No distinctly aberrant population consistent with a T-LGL leukemia was identified". The postsplenectomy CBC white count shows resolution of the leukopenia and neutropenia and further supports the likely benign T-cell population. The patient's neutropenia has resolved post splenectomy without any lymphocytosis or evidence of underlying malignancy followed up to nine months following splenectomy. The reoccurring bouts of severe abdominal pain, recurrent infections from neutropenia and standard of living have significantly improved after her splenectomy.

\section{Learning Points/Take Home Messages}

- Splenomegaly can arise from several etiologies including chronic inflammation as was suspected in our patient with RA.

- Felty's syndrome was seen in our patient with non-optimal disease modifying antirheumatic treatment.

- Patients with recurrent infections and neutropenia, splenectomy should be considered.

- Splenectomy in patients with various hematologic has been associated with an increased incidence of vascular complications, including venous and arterial thrombosis.

- Malignancy must be ruled out following massive splenomegaly in association with severe haematological disorders.

\section{References}

1. Hatch FN (1945) Atrophic arthritis associated with splenomegaly and leukopenia. Ann Intern Med 23: 201-220.

2. Campion G, Maddison PJ, Goulding N, James I, Ahern MJ, et al. (1990) The Felty syndrome: a case-matched study of clinical manifestations and outcome, serologic features, and immunogenetic associations. Medicine (Baltimore) 69: 69-80.

3. Bickenbach KA, Gonen M, Labow DM, Strong V, Heaney ML, et al. (2013) Indications for and efficacy of splenectomy for haematological disorders. $\mathrm{Br}$ J Surg 100: 794-800.

4. Laszlo J, Jones R, Silberman HR, Banks PM (1978) Splenectomy for Felty's syndrome. Clinicopathological study of 27 patients. Arch Intern Med 138: 597602.

5. Khan PN, Nair RJ, Olivares J, Tingle LE, Li Z (2009) Postsplenectomy reactive thrombocytosis. Proc (Bayl Univ Med Cent) 22: 9-12.

6. Logan MS, Watson CM, Nottingham JM (2009) Symptomatic splenomegaly in polycythemia vera: a review of the indications for splenectomy and perioperative considerations. Am Surg 75: 363-368.

7. O'Reilly RA (1998) Splenomegaly in 2,505 patients at a large university medical center from 1913 to 1995.1963 to $1995: 449$ patients. West J Med 169: 88-97.

8. Alba D, Diaz Lobato S, García-Quintero T, Arribas P (1996) Splenothoracic fistula complicating primary splenic hydatidosis. J Thorac Cardiovasc Surg 111: 1103-1104.

9. Kojodjojo P, Chew S, Wakeham K, Lancaster R (2005) Splenomegaly, anaemia and pleural effusion. J R Soc Med 98: 23-24.

10. Dendle C, Spelman T, Sundararajan V, Chunilal S, Woolley I (2015) An analysis of the thromboembolic outcomes of 2472 splectomized individuals. Blood 125: 1681-1682.

11. Morgenstern L, Skandalakis JE (1997) Anatomy and embryology of the spleen. In: Hiatt JR, Phillips EH, Morgenstern L (Eds), Surgical diseases of the spleen. Springer-Verlag, Berlin/Heidelberg, pp. 15-24. 